Revue d'histoire de l'Amérique française

REVUE D.HISTOIRE DE L'AMÉRIQUE FRANÇAISE

\title{
Carrières et vie matérielle du clergé du Richelieu-Yamaska (1790-1840)
}

\section{Christine Hudon}

Volume 45, numéro 4, printemps 1992

URI : https://id.erudit.org/iderudit/305018ar

DOI : https://doi.org/10.7202/305018ar

Aller au sommaire du numéro

Éditeur(s)

Institut d'histoire de l'Amérique française

ISSN

0035-2357 (imprimé)

1492-1383 (numérique)

Découvrir la revue

Citer cet article

Hudon, C. (1992). Carrières et vie matérielle du clergé du Richelieu-Yamaska (1790-1840). Revue d'histoire de l'Amérique française, 45(4), 573-594.

https://doi.org/10.7202/305018ar
Résumé de l'article

À la fin du XVIII ${ }^{\mathrm{e}}$ et au début du XIX ${ }^{\mathrm{e}}$ siècle, l'Église bas-canadienne est confrontée à une pénurie de prêtres. Les carrières cléricales en subissent les contrecoups. Les vicariats sont généralement courts et les promotions, rapides. Les effets de cette situation se font aussi sentir sur la vie matérielle du clergé. Les paroisses, souvent vastes et populeuses, procurent aux curés des revenus substantiels. La desserte d'une ou de plusieurs communautés voisines permet d'augmenter les gains en cumulant les dîmes. Ces revenus confèrent aux curés une fortune supérieure à celles des membres des autres groupes socioprofessionnels, à l'exception des grands marchands. 


\title{
CARRIÈRES ET VIE MATÉRIELLE DU CLERGÉ DU RICHELIEU-YAMASKA $(\mathbf{1 7 9 0}-1840)^{1}$
}

\author{
CHRISTINE HUDON \\ Centre d'études québécoises \\ Université du Québec à Trois-Rivières
}

\section{RÉSUMÉ}

À la fin du XVIII ${ }^{e}$ et au début du XIX ${ }^{e}$ siècle, l'Église bas-canadienne est confrontée à une pénurie de prêtres. Les carrières cléricales en subissent les contrecoups. Les vicariats sont généralement courts et les promotions, rapides. Les effets de cette situation se font aussi sentir sur la vie matérielle du clergé. Les paroisses, souvent vastes et populeuses, procurent aux curés des revenus substantiels. La desserte d'une ou de plusieurs communautés voisines permet d'augmenter les gains en cumulant les dîmes. Ces revenus confèrent aux curés une fortune supérieure à celles des membres des autres groupes socioprofessionnels, à l'exception des grands marchands.

\section{ABSTRACT}

At the end of the eighteenth and the beginning of the nineteenth centuries, the Lower Canada church faced a shortage of priests. This affected clerical careers in several ways. Clerics spent but a brief time as vicars and were promoted rapidly. The often vast and populous parishes generated substantial revenues for the curés. Priest serving and drawing tithes from two parishes could increase these gains even more. Such revenues permitted the curés to amass fortunes greater than those of the members of all other socio-professional groups, the richest merchants excepted.

\section{INTRODUCTION}

Acteur social d'importance, le prêtre a déjà suscité plusieurs études. Parmi celles-ci, l'article pionnier de Louis-Edmond Hamelin a mis en évidence la pénurie d'effectifs cléricaux des décennies

1 Cet article constitue la version remaniée d'une partie de mon mémoire de maîtrise, Les curés du Richelieu-Yamaska, 1790-1840. Recrutement, vie matérielle et action pastorale, Université de Montréal, 1990, $158 \mathrm{p}$. Une bourse du fonds FCAR a rendu possible cette recherche. Je remercie mes directeurs, Christian Dessureault et Jean Roy, pour leurs judicieux conseils. Merci également à Serge Gagnon qui a commenté ce texte.

RHAF, vol. 45, nº 4, printemps 1992 
subséquentes à la Conquête ${ }^{2}$. Le ratio fidèles/prêtre, estimé à 500 pour 1 en 1760, excédait 1800 pour 1 vers $1830^{3}$. De nombreuses paroisses étaient alors privées d'un curé résidant. Le surmenage et la maladie guettaient les prêtres chargés de la desserte de plusieurs communautés ${ }^{4}$. La formation ecclésiastique subit aussi les contrecoups de cette situation. Afin de doter les paroisses d'un pasteur, les évêques eurent tendance à réduire la durée des études théologiques ${ }^{5}$. En ce qui concerne l'origine du clergé, Louise et Serge Gagnon ont remarqué, pour la même époque, un recrutement de plus en plus tourné vers les campagnes, avec un apport croissant de la paysannerie ${ }^{6}$.

Les travaux des trente dernières années ont donc contribué à circonscrire le profil du clergé de la fin du XVIII et du début du XIX ${ }^{e}$ siècle. Certaines facettes de la vie des prêtres restent cependant méconnues. Par exemple, les carrières et les revenus ecclésiastiques ont peu retenu l'attention. Soucieuse d'édifier le lecteur, l'histoire religieuse de type hagiographique s'est surtout attardée à l'aspect spirituel des carrières ecclésiastiques, en négligeant les questions matérielles. L'ambition cléricale ne pouvait non plus trouver place dans ces études. Plusieurs travaux sur la socio-économie des paroisses ont cependant pris en compte les revenus curiaux ${ }^{7}$. L'image d'un clergé bien pourvu se dégage de cette production. Par une étude circonscrite à quatorze paroisses du Richelieu-Yamaska, nous souhaitons

2 Louis-Edmond Hamelin, «Evolution numérique séculaire du clergé catholique dans le Québec», Recherches sociographiques, 2,2 (avril-juin 1961): 189-241.

3 Il s'abaissa dans les décennies suivantes pour atteindre un prêtre pour 510 fidèles en 1880. Voir L.-E. Hamelin, loc. cit., 238.

4 Voir Jean-Pierre Wallot, «La religion catholique et les Canadiens au début du XIX ${ }^{\circ}$ siècle», Un Québec qui bougeait. Trame socio-politique au tournant du XIX` siècle (Sillery, Boréal Express, 1973), 191-198.

5 Voir notamment Pierre Savard, "La vie du clergé québécois au XIXe siècle», Recherches sociographiques, 8,3 (septembre-décembre 1967): 262; James Harold Lambert, Monseigneur The Catholic Bishop. Joseph-Octave Plessis, Church, State and Society in Lower Canada: Historiography and Analysis, thèse de doctorat (histoire), Université Laval, 1981, 509; Lucien Lemieux, Histoire du catholicisme québécois. Les XVIII' et XIX'siècles, tome 1: Les années difficiles (Montréal, Boréal, 1989), 113-114.

6 Louise Lebel-Gagnon et Serge Gagnon, «Le milieu d'origine du clergé québécois, 1775-1840: mythes et réalités», Revue d' histoire de l'Amérique française, 37,3 (décembre 1983): 373-393.

7 Voir Allan Greer, Peasant, Lord, and Merchant. Rural Society in Three Quebec Parishes 1740-1840 (Toronto, University of Toronto Press, 1987), 114; pour la seconde moitié du XIX ${ }^{e}$ siècle, voir Normand Séguin, La conquête du sol au XIX` siècle (Sillery, Boréal Express, 1977), 189-191. Richard Chabot et Fernand Ouellet ont ausi souligné l'importance des revenus curiaux. R. Chabot, Le curé de campagne et la contestation locale au Québec de 1791 aux troubles de 1837-38 (Montréal, Hurtubise HMH, 1975), 129; F. Ouellet, Histoire économique et sociale du Québec, 1760-1850. Structures et conjonctures (Montréal, Fides, 1971), 2 vol., et Le Bas-Canada, 1791-1840. Changements structuraux et crises (Ottawa, Éditions de l'Université d'Ottawa, 1976), 65-67. 
ajouter quelques éléments pour une sociographie du clergé, en examinant l'influence de la crise des vocations sur le cheminement professionnel et les revenus des prêtres. Ces deux aspects de la vie cléricale méritent d'être traités ensemble, car maintes promotions s'exprimaient par une nomination à une cure plus lucrative. Une première partie présentera les principales étapes de la carrière pastorale. Nous verrons comment la pénurie de prêtres s'est reflétée sur le déroulement des carrières. Une seconde partie traitera du statut économique du clergé paroissial. Après un examen des revenus curiaux des paroisses du Richelieu-Yamaska, nous présenterons les principales interventions économiques des curés et comparerons leur niveau de fortune à celui des autres groupes socio-professionnels.

Les quatorze paroisses du Richelieu-Yamaska présentent entre elles plusieurs dissemblances qui permettront d'examiner les cheminements de carrière, certaines places étant très recherchées, d'autres comportant peu d'attraits. La région est ainsi constituée de sols argileux très fertiles, mais elle contient aussi certains terroirs moins favorables aux cultures céréalières. La paroisse Saint-Césaire et les rangs Salvail et Les Étangs, à La Présentation, appartiennent à cette seconde catégorie. Le degré variable du développement des terroirs contribue également à la diversité régionale. À mesure que les seigneuries se peuplèrent, des missions ${ }^{8}$ furent fondées. Entre 1790 et 1840, de nouvelles unités religieuses se greffèrent aux paroisses plus anciennes de Saint-Denis, Saint-Charles et Saint-Antoine, constituées au milieu du XVIII ${ }^{e}$ siècle, et à celles de Belœil et de Saint-Hyacinthe, respectivement fondées en 1772 et 1777.

Dans le Richelieu-Yamaska, la charge pastorale par prêtre était particulièrement lourde. En 1790, elle s'élevait à 1840 paroissiens; en 1823, elle excédait 2000 fidèles $^{9}$. Parmi les paroisses les plus populeuses, Saint-Hyacinthe comptait 6552 habitants en 1823; SaintDenis, Saint-Jean-Baptiste et Saint-Antoine comprenaient respectivement 2724,2154 et 1933 personnes. Les membres du corps sacerdotal avaient donc des responsabilités imposantes. Les carrières cléricales s'en voyaient bouleversées.

8 Les missions sont des unités religieuses qui ont ouvert leurs registres mais qui n'ont pas été érigées officiellement. Elles sont nombreuses à la fin du XVIII ${ }^{e}$ et au début du XIX ${ }^{e}$ siècle car, après la Conquête, l'Église catholique du Québec perdit le droit d'ériger de nouvelles paroisses. Grâce à un contexte plus favorable, plusieurs érections furent promulguées à compter de 1825.

9 Archives de la chancellerie de l'Archevêché de Montréal (ACAM), RC, 1, État des paroisses visitées en 1823 . 


\section{1 - LA CARRIÈRE PASTORALE}

\section{Le clergé paroissial du Richelieu-Yamaska}

Peu de temps après son ordination, parfois le jour même, le prêtre était généralement nommé vicaire. Assigné aux tâches secondaires et souvent ingrates, il faisait alors son premier apprentissage du ministère paroissial. Mgr Lartigue prescrivait aux vicaires «d'épargner à Mr. le Curé les fonctions pénibles telles que la visite des malades dans les mauvais temps et l'instruction particulière des Ignorants ${ }^{10}$ ». Les jeunes clercs se devaient de faire bonne figure auprès de leur patron, car un rapport défavorable de celui-ci avait des conséquences fâcheuses sur les nominations subséquentes. En plus d'être soumis à un prêtre parfois très exigeant, ils résidaient avec la ménagère et, dans plusieurs cas, avec les parents du curé. Des conflits risquaient de surgir. À Saint-Denis, les sœurs du curé avaient rendu le presbytère «inaccessible aux voisins et pas tenable pour les vicaires ${ }^{1{ }^{11}}$ ». Cette première étape de la carrière cléricale s'avérait toutefois relativement courte. La plupart des 46 prêtres qui furent vicaires dans le RichelieuYamaska, ne le demeurèrent que quelques mois. Le mandat moyen dura près de deux années et demie, mais deux vicariats de 9 et 14 ans gonflent cette moyenne. Ces cas extrêmes écartés, celle-ci s'établit à un peu moins de 2 ans.

L'immense paroisse de Saint-Hyacinthe, d'où furent successivement retranchées La Présentation, Saint-Damase, Saint-Césaire, SaintPie, Sainte-Rosalie et Saint-Dominique, accueillit le plus grand nombre de vicaires. À partir du début du XIX ${ }^{\mathrm{e}}$ siècle, le curé maskoutain bénéficia toujours de l'aide d'un, voire de deux ou de trois auxiliaires. En plus de vaquer au ministère paroissial, les jeunes ecclésiastiques assumaient fréquemment diverses fonctions au collège fondé en 1811 . À plusieurs reprises, le curé de Saint-Denis profita aussi des services d'un jeune prêtre. Dans la plupart des paroisses, la présence d'un vicaire était toutefois exceptionnelle ${ }^{12}$. À l'occasion, l'un d'eux épaulait un curé malade, âgé ou simplement responsable d'un grand territoire. Pierre Robitaille, aumônier des troupes du Sud du BasCanada entre 1812 et 1815 , et desservant les paroisses Saint-Charles

10 ACAM, 295.101, 829-61, Règlement de vie pour les vicaires, 1829.

11 Archives de l'Évêché de Saint-Hyacinthe (AESH), XVII, c.25, lettre de Demers à Lartigue, 19 septembre 1836.

12 En 1833, le diocèse de Québec, qui couvrait alors tout le Bas-Canada et le district épiscopal du Nord-Ouest, ne comptait que 44 vicaires. Seulement 17 d'entre eux officiaient dans le district de Montréal, ce qui faisait dire à Mgr Lartigue que son district était nettement défavorisé. ACAM, RLL, 7, 186, lettre de Lartigue à Signay, 31 juillet 1833. 
et Saint-Marc, fut ainsi secondé par un vicaire. Plusieurs curés désignés pour biner dans une communauté voisine n'eurent cependant pas cette chance. Certains durent desservir, sans aucun secours, de 2000 à 3000 habitants. Tel fut le lot des titulaires de la cure de Belœil, aussi chargés de la mission de Saint-Hilaire.

Les vicaires étaient donc peu nombreux dans le RichelieuYamaska et plusieurs curés assumèrent seuls la desserte de leurs paroisses. Attardons-nous au cheminement professionnel de ces derniers. Afin de le reconstituer, nous avons fait appel au dictionnaire Allaire $^{13}$ et à la correspondance cléricale, qui ont fourni les assignations successives ${ }^{14}$ et les raisons des mutations de chacun des 49 curés qui desservirent les 14 paroisses.

\section{Configuration générale des carrières}

La majorité des nominations coïncidaient avec la Saint Michel (29 septembre), qui marquait le début d'une nouvelle année pour la perception des dîmes. Pour l'évêque, les mutations étaient une source de problèmes. Il devait pourvoir aux besoins du diocèse et récompenser les prêtres méritants, sans pour autant froisser quiconque. Avec la pénurie d'effectifs cléricaux, cet exercice s'avérait un réel cassetête. Les contraintes d'un personnel pastoral insuffisant se répercutèrent sur la configuration générale de la carrière des prêtres en accélérant leur avancement professionnel. Le schéma d'une carrière ascendante ${ }^{15}$, où le prêtre exerçait d'abord le vicariat, puis dirigeait une ou plusieurs petites paroisses, avant d'obtenir une cure de grande envergure, comptait plusieurs exceptions à la fin du XVIII ${ }^{e}$ et au début du XIX ${ }^{\mathrm{e}}$ siècle.

Plus de la moitié des 49 prêtres ${ }^{16}$ furent nommés curés après trois ans ou moins de vicariat (tableau 1). Six d'entre eux sautèrent même cette étape et furent affectés à une cure dans les jours qui suivirent leur ordination. Les évêques préféraient alors doter les paroisses d'un curé résidant plutôt que de multiplier les vicariats. Promus au sacerdoce

13 Jean-Baptiste-Arthur Allaire, Dictionnaire biographique du clergé canadien-français. Les Anciens (Montréal, Imprimerie de l'École Catholique des Sourds-Muets, 1910), 543 p. Cet ouvrage comporte toutefois des lacunes et des erreurs qu'il faut rectifier en recourant aux archives diocésaines.

14 À ce sujet, voir Jean Roy, «Le clergé nicolétain, 1885-1904: aspects sociographiques», RHAF, 35,3 (décembre 1981): 383-395.

15 Voir L. Lemieux, op. cit., 139-140. En France, la carrière du clergé paroissial du $\mathrm{XIX}^{\mathrm{c}}$ siècle passe, en gros, par les mêmes étapes. Voir notamment Marcel Launay, Le bon prêtre. Le clergé rural au XIX' siècle (Paris, Aubier, 1986), 86-87. de Nancy.

16 Tous sont d'origine canadienne, à l'exception d'Antoine-Joseph Ginguet, du diocèse 
TABLEAU 1

Intervalle entre l'ordination et l'obtention de la première cure 1760-1840*

\begin{tabular}{|c|r|}
\hline Moins de 1 an & 9 \\
Entre 1 et 2 ans & 10 \\
Entre 2 et 3 ans & 6 \\
Entre 3 et 4 ans & 13 \\
Entre 4 et 5 ans & 5 \\
Entre 5 et 6 ans & 3 \\
Entre 6 et 7 ans & 1 \\
Plus de 7 ans & 1 \\
\hline
\end{tabular}

* Certains curés furent nommés avant 1790

vers l'âge de 24 ans, la majorité des 49 prêtres obtinrent leur premier mandat curial à un âge assez précoce: trente-quatre d'entre eux (près des trois quarts) n'avaient pas 30 ans. La jeunesse de ce clergé n'était pas sans incidence sur les relations qu'il établissait avec les paroissiens. Sans doute était-il dynamique et enthousiaste, mais l'inexpérience lui faisait commettre certaines bourdes. Il semble également que les habitants d'âge mûr toléraient mal de se faire diriger par de jeunes prêtres. L'un d'eux, Pierre Lafrance, 25 ans, considéra, à son arrivée à Saint-Jean-Baptiste de Rouville, que le presbytère était trop exigu. Il s'empressa alors de convoquer les paroissiens pour leur faire part de ses exigences, puis donna ordre à des ouvriers d'agrandir la maison curiale en rognant l'espace sur la salle des habitants. Ces procédés eurent tôt fait de mécontenter les paroissiens qui se plaignirent à l'évêque. Celui-ci blâma sévèrement le curé de son manque de $\operatorname{tact}^{17}$. Au delà des questions d'administration paroissiale, l'arrivée de jeunes prêtres, à la tête des paroisses, dut se répercuter sur l'activité pastorale et le type d'encadrement dispensé. Ces questions mériteraient d'être étudiées.

Par une promotion rapide à une cure d'importance, des ecclésiastiques s'éloignaient encore du cheminement professionnel type, sensé être plus graduel. L'assignation à une grosse cure n'était pas uniquement affaire d'expérience. Quand une place se libérait, l'autorité épiscopale pouvait y appeler un jeune prêtre, s'il le jugeait plus capable. Jean-Baptiste Bélanger fut ainsi rapidement promu à un poste 
d'importance. Après avoir œuvré au collège de Saint-Hyacinthe, il fut nommé curé de la prospère paroisse de Belœil, trois ans seulement après son ordination. Les promotions rapides créaient des tensions au sein du clergé. Un curé d'expérience, convaincu d'avoir été victime d'une injustice, écrivait à Mgr Plessis ${ }^{18}$ :

Vous devez vous rappeler, Monseigneur, que l'orsqu'à Berthier, vous m'avez accordé audience, j'ai demandé à votre Grandeur, s'il lui plaisoit remplir la promesse qu'elle m'avoit faite de me donner une cure dans le district de Québec aussitôt qu'un vuide auroit lieu: vous m'avez répondu que vous n'avez pas de cure \& cependant le Cap Santé étoit et est encore vaquant: mais cette cure est promise au jeune Mr Painchaud ${ }^{19}$ comme précédemment St. Denis à $\mathrm{Mr}$ Kelley ${ }^{20} \&$ Larivière du loup au jeune Mr Lebourdais $^{21}$. Il faut avouer monseigneur que nous sommes dans un siècle extraordinaire qui enfante sans efforts les plus grands sujets puisque par votre conduite l'on doit juger que la sagesse, la science \& l'expérience sont presque innés dans les plus jeunes sujets de votre clerger ${ }^{22}$.

Pour éviter de déplaire aux prêtres plus âgés, l'évêque pouvait se livrer à diverses tractations. La correspondance échangée entre les autorités épiscopales et certains curés en 1820 et 1821 en fournit un exemple. La cure de Belœil était alors vacante, à la suite de la mort de son titulaire, l'abbé Prévost. Mgr Plessis offrit la place à un curé d'expérience, persuadé que celui-ci refuserait et qu'il serait alors possible de la donner «à un jeune prêtre, sans faire crier les plus anciens ${ }^{23}$ ». L'évêque fut cependant pris au mot: le curé accepta son offre. Il demanda alors à un prêtre des environs, l'abbé Robitaille, de s'enquérir des dispositions des Belœillois à l'égard du candidat à la cure. Les paroissiens devaient savoir que l'aspirant au poste prévoyait «faire beaucoup de réparations aux dépendances du presbytère» et qu'il faudrait y pourvoir par des contributions ${ }^{24}$. Ainsi informés des

18 Dans cet article, nous reproduisons des extraits de la correspondance cléricale, sans en corriger l'orthographe ni la ponctuation.

19 Celui-ci, alors missionnaire dans la Baie-des-Chaleurs, n'ira jamais à Cap Santé. En 1814, il devint curé de Sainte-Anne de La Pocatière. À l'époque, les évêques souhaitaient récompenser les missionnaires afin «d'encourager les jeunes prêtres à faire (...) quelques années de mission pénible». AAQ, RL, 2, 364, lettre de Denaut à Plessis.

20 Jean-Baptiste Kelly, nommé curé de Saint-Denis à l'âge de 27 ans, avait toute la confiance de Mgr Plessis. Il avait été son secrétaire, pendant ses études, entre 1803 et 1806.

21 Celui-ci était le neveu de Mgr Panet, coadjuteur de l'évêque. Il fut placé à Louiseville en 1813 , à 30 ans.

22 AESH, XVII, c.41, lettre de Germain à Plessis, 9 mars 1814. Charles-François Germain avait alors 41 ans.

23 AAQ, RL, 10, lettre de Plessis à Lartigue, 3 mars 1821.

24 AAQ, RL, 10, lettre de Plessis à Robitaille, 14 mars 1821. 
intentions - réelles ou supposées - de leur futur curé, les habitants de Belœil se montrèrent peu disposés à l'accueillir ${ }^{25}$. André-Toussaint Lagarde, un prêtre de 29 ans, obtint la cure.

Tous les prêtres ne recherchaient cependant pas un bénéfice très lucratif ou des responsabilités importantes. La timidité, un manque de confiance en soi, une santé déficiente poussaient quelques curés à demander une paroisse de petite taille ou à refuser une promotion. En 1832, Amable Brais, curé de Saint-Pie, sollicitait ainsi une paroisse moins populeuse en raison de sa faible santée ${ }^{26}$.

Le modèle idéal de la carrière pastorale ascendante souffrait encore une exception, la «disgrâce ${ }^{27}$ », perçue comme un déshonneur pour le prêtre qui en était frappé, comme pour tout le corps sacerdotal. À la suite d'un comportement répréhensible, des ecclésiastiques étaient mutés à une cure de moindre importance, à une mission en périphérie ou à un vicariat. Dans certains cas, une suspension temporaire ou définitive des fonctions sacerdotales sanctionnait la conduite déviante. Un «règlement de vie», qui imposait un emploi du temps très serré, était attribué aux prêtres démis.

La surveillance exercée par les paroissiens ${ }^{28}$ et par les confrères prêtres $^{29}$ permettait de repérer rapidement les clercs qui avaient manqué à la discipline ecclésiastique. L'autorité cléricale veillait quant à elle à étouffer le scandale et à punir les prêtres coupables. Les motifs les plus fréquents de rétrogradation étaient l'insubordination ${ }^{30}$, l'alcoolisme ${ }^{31}$ et les écarts à la morale sexuelle ${ }^{32}$. Il s'avérait difficile,

25 AAQ, RL, 10, lettre de Plessis à Lajus, 17 avril 1821.

26 AAQ, RL, 15, lettre de Panet à Brais, 17 novembre 1832. Voir aussi AESH, XVII, c.62, lettre de Duguay à Panet, 3 juillet 1819 .

27 Ce terme revient fréquemment dans la correspondance cléricale pour désigner les mutations défavorables. Philippe Boutry en a également remarqué l'usage dans le diocèse de Belley, au XIX' siècle. Voir Prêtres et paroisses au pays du Curé d' Ars (Paris, Cerf, 1986), 274.

28 AAQ, RL, 17, 439, lettre de Cazeau à M. D. Meunier, 20 août 1836 et ACAM, RLL, 8, 209, lettre de Lartigue à Naud, 9 juillet 1836. Des requêtes demandant le rappel de certains prêtres sont contenues dans les dossiers de paroisse aux AESH.

29 Une lettre de l'abbé Charles LaRocque à Mgr Lartigue en fournit un exemple. AESH, XVII, c.65, 24 décembre 1838. Les AESH conservent aussi cette supplique d'un curé convaincu d'être victime des ragots de certains collègues: «je vous conjure de ne point écouter ni croire les rapports de gens mal intentionnés et peut-être jaloux, je ne sais de quoi.»XVII, c.25, Bédard à Plessis, 17 mars 1819.

30 Le curé Naud de Saint-Jean-Baptiste est sans doute le prêtre récalcitrant le plus connu. En 1836, il refusa de quitter la paroisse de Saint-Jean-Baptiste, puis intenta un procès à son successeur et à l'évêque de Montréal. Voir J.-J. Lefebvre, «Le curé Louis Naud (circa 17991843)», SCHEC, Rapport (1956-1957): 65-90.

31 Ainsi, les abbés F.-L. Arsenault et R.-F. Lajus, dont les longs vicariats de 9 et 14 ans ont été évoqués plus haut, avaient encouru la désapprobation de l'évêque pour leur propension à s'enivrer.

32 Voir Serge Gagnon, Plaisir d'amour et crainte de Dieu. Sexualité et confession au Bas-Canada (Québec, Presses de l'Université Laval, 1991), 21-29. 
pour les ecclésiastiques déchus, de se conformer à une nouvelle vie où leurs fonctions, leur prestige et leurs pouvoirs étaient considérablement amoindris. L'un d'eux, J.-A. Boisvert, multiplia les lettres à Mgr Bourget pour lui rappeler que «la position de subalterne était tout à fait contraire à [s]on caractère ${ }^{33}$. En 1836 , il avait été destitué de la cure de Saint-Pie, à la suite d'une enquête faisant état de ses abus d'alcool. Nommé vicaire, il s'enfuit de son poste «après avoir été ivre jusqu'à l'autel y dire la messe et (...) avoir fait des vacarmes horri-

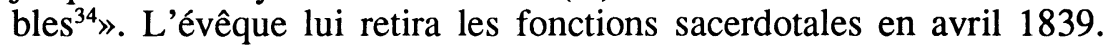
Dès le mois d'octobre, il put exercer de nouveau, mais demeura vicaire jusqu'en 1850, malgré ses appels incessants pour obtenir une cure. Redevenir vicaire et devoir obéir à un confrère, parfois plus jeune, était donc difficile. Tous n'avaient pas le franc-parler de l'abbé Boisvert, mais, dans un style plus larmoyant et prodigue en bons mots, d'autres prêtres repentants implorèrent également la clémence de l'évêque ${ }^{35}$.

Si le cheminement professionnel de certains ecclésiastiques fut marqué par une rétrogradation causée par leur conduite répréhensible, la pénurie de prêtres hâta, pour le plus grand nombre, les promotions. Les mutations étaient alors nombreuses. La plupart des curés du Richelieu-Yamaska demeurèrent dans la même paroisse 10 années ou moins. Dix-huit mandats excédèrent tout de même 10 ans (tableau 2). En général, les cures très lucratives connaissaient des ministères d'une plus grande stabilité. Les retraites étant rares, la majorité des curés dirigeaient une paroisse jusqu'à leur décès. Avant 1840, seulement cinq prêtres du Richelieu-Yamaska se retirèrent du ministère ${ }^{36}$. La stabilité des mandats était donc liée à divers facteurs, notamment à l'importance des revenus. La hiérarchie des cures, que nous avons déjà évoquée, mérite ici d'être examinée de plus près.

\section{2 - LE STATUT ÉCONOMIQUE DU CLERGÉ}

\section{Stabilité des ministères et rentabilité des cures}

Toutes les cures n'exerçaient pas le même attrait. En raison de l'éloignement, de la pauvreté qui y sévissait souvent et de l'édifice cultuel à parachever, bien des prêtres se rendaient avec regret dans une communauté récemment fondée. À l'opposé, une paroisse, «avanta-

33 ACAM, 420.020, 845-2, lettre de Boisvert à Bourget, 21 août 1845.

34 ACAM, RLL, 9, 39, lettre de Lartigue à Boisvert, avril 1839.

35 Par exemple, AESH, XVII, c.11, lettre de Gaillard à Plessis, 14 juin 1806.

36 Ce nombre s'abaisse encore si l'on considère que deux des cinq curés avaient été destitués à vie par une sanction disciplinaire. 
TABLEAU 2

Durée des cures dans les paroisses du Richelieu-Yamaska 1790-1840*

\begin{tabular}{|c|c|}
\hline Moins de 1 an & 1 occurence \\
1 à 5 ans & 23 occurences \\
6 à 10 ans & 11 occurences \\
11 à 15 ans & 4 occurences \\
16 à 20 ans & 6 occurences \\
21 à 25 ans & 3 occurences \\
26 à 30 ans & 4 occurences \\
31 à 35 ans & - \\
36 à 40 ans & 1 occurence \\
\hline
\end{tabular}

- Le nombre total de ministères excède 49 car des prêtres furent curés de plusieurs paroisses du Richelieu-Yamaska.

geuse, par sa situation, par son excellent revenu, par la paix qui y régn[ait], et par le peu d'étendue de son territoire, quoiqu'assez peuplée $^{37} \gg$ faisait envie. À moins d'être malades ou d'éprouver des difficultés dans leurs rapports avec les paroissiens, les titulaires de semblables cures demandaient rarement une mutation. Saint-Charles, Saint-Antoine et Saint-Denis constituaient notamment des places recherchées. Établies vers 1750 , ces paroisses fertiles étaient bien développées au tournant du XIX ${ }^{\mathrm{e}}$ siècle. Aussi ont-elles conservé longtemps leurs curés. La première fut desservie par le même prêtre pendant 20 ans, tandis que les curés de Saint-Antoine et de SaintDenis exercèrent des mandats d'une durée moyenne de 18 et de 23 ans. Très différente fut la situation des desservants de Saint-Césaire, fondée en 1822. Leur séjour à la tête de cette paroisse fut d'une durée moyenne de 3 ans, car tour à tour ils demandèrent à être mutés à un poste plus rémunérateur ${ }^{38}$. De fait, la paroisse Saint-Césaire, aussi surnommée Sainte-Misère ${ }^{39}$, était très pauvre ${ }^{40}$ et peu lucrative pour

37 AAQ, RL, 2, 148, lettre de Hubert à Labadie, 13 septembre 1794.

38 ACAM, RL, 2, 150, lettre de Lartigue à Plessis, 5 mars 1823; AAQ, RL, 11, 178, lettre de Plessis à Lartigue, 22 avril 1823; AESH, XVII, c.18, lettre de Poirier à Plessis, 13 avril 1823; ACAM, RLL, 6, 75, lettre de Lartigue à Delisle, 17 septembre 1831.

39 Isidore Desnoyers, Histoire de la paroisse de Saint-Damase, document manuscrit conservé aux AESH, 33.

40 Sur la pauvreté des paysans de Saint-Césaire, voir Christian Dessureault, «Crise ou modernisation? La société maskoutaine durant le premier tiers du $\mathrm{XIX}^{\circ}$ siècle», RHAF, 42,3 (hiver 1989): 382-383. 
les curés. Les relevés des dîmes et du casuel vont permettre d'apprécier les revenus qu'elle générait et de les comparer à ceux des autres paroisses.

\section{Les revenus des curés}

La dîme, vingt-sixième partie de la récolte de céréales et de pois de chaque habitant, constituait l'essentiel du revenu curial. À cela s'ajoutait le casuel, perçu par le curé et par la fabrique, pour la célébration des messes, des mariages et des sépultures.

La dîme se comptait d'une Saint Michel à l'autre, mais n'était payable qu'au temps pascal. Ce délai permettait aux habitants d'exécuter le battage des grains, un travail long et fort exténuant. Les tableaux 3 et 4 donnent un aperçu des recettes produites par les dîmes vers 1788-1789 et vers 1834 . Ces chiffres constituent des moyennes approximatives du revenu des années qui précédaient la visite pastorale ${ }^{41}$. Ils mettent en évidence les différences entre les paroisses. L'ancienneté des terroirs, la qualité des sols, l'importance de la population et le type de culture pratiquée influençaient la rentabilité des cures. Vers 1789 comme dans les années 1830, Belœil rapportait ses 1000 minots de blé, alors que certaines paroisses, comme La Présentation et surtout Saint-Césaire, procuraient un revenu beaucoup moins important. La faiblesse des revenus de cette dernière cure préoccupait beaucoup les évêques Plessis et Lartigue ${ }^{42}$.

Dans les paroisses pauvres comme Saint-Césaire, l'évêque pouvait exiger le paiement d'une somme fixe - habituellement $£ 100^{43}$ - ou de 400 minots de blé, afin d'assurer un revenu «honorable» au curé. À défaut de remplir cette charge, la communauté était privée d'un curé résidant. Pendant quelques années, la paroisse Saint-Césaire fut ainsi jumelée à celle de Saint-Damase. Dans certaines paroisses, une fraction des cultures secondaires était exigée. Les habitants de SaintHilaire durent par exemple s'engager à payer le vingt-sixième des pommes récoltées, une culture normalement exempte de toutes redevances $^{44}$. Les revenus curiaux sur ce produit furent toutefois minimes,

41 Thomas Wien, «Visites paroissiales et production agricole au Canada vers la fin du XVIII' siècle», François Lebrun et Normand Séguin, Sociétés villageoises et rapports villescampagnes au Québec et dans la France de l'Ouest XVII ${ }^{e}-X X^{e}$ siècles (Trois-Rivières, Université du Québec à Trois-Rivières, 1987), Actes du colloque franco-québécois (1985), 184.

42 AAQ, RL, 11, lettre de Plessis à Lartigue, 24 avril 1823; ACAM, RLL, 2, 150 et 88, lettres de Lartigue à Delisle, 17 et 29 septembre 1831.

43 Soit 2400 livres de 20 sols, la livre anglaise équivalant à 24 livres ancien cours.

44 AESH, XVII, c.35, lettre de Rouville à Panet, 24 août 1830. Au sujet de la dîme sur les récoltes secondaires, voir Thomas Wien, loc. cit., 186. 


\section{TABLEAU 3}

Dîmes sur les principaux produits dans les paroisses du Richelieu Yamaska vers $1788-1789$ (en minots)*

\begin{tabular}{lcccrr}
\hline \multicolumn{1}{c}{ Paroisse } & $\begin{array}{c}\text { Date de } \\
\text { fondation }\end{array}$ & Blé & Avoine & Pois & Population \\
\hline Saint-Hyacinthe & 1777 & 500 & 100 & 80 & 1360 \\
Saint-Charles & 1740 & 850 & 150 & 60 & 1324 \\
Saint-Antoine & 1750 & 1000 & 200 & 60 & 1285 \\
Saint-Denis & 1740 & 1100 & 400 & 100 & 1694 \\
Beloeil & 1772 & 1000 & 400 & 120 & 1702 \\
\hline
\end{tabular}

Sources: AAQ, 69 CD, vol.1; recensement de 1790. Reproduit dans Thomas Wien, loc. cit., 190. - Les quatre premières paroisses ont été visitées en 1788. Beloeil a reçu l'évêque l'année suivante.

\section{TABLEAU 4}

Les dîmes sur les principaux produits dans les paroisses du Richelieu-Yamaska vers 1834 (en minots)

\begin{tabular}{lcccrc}
\hline \multicolumn{1}{c}{ Paroisse } & $\begin{array}{c}\text { Date de } \\
\text { fondation }\end{array}$ & Blé & Avoine & Pois & $\begin{array}{r}\text { Population } \\
\text { catholique } \\
\text { en 1831 }\end{array}$ \\
\hline Saint-Césaire & 1822 & 160 & 60 & 25 & 2435 \\
La Présentation & 1806 & 300 & 150 & 40 & 1646 \\
Saint-Hilaire & 1799 & 300 & 150 & 100 & 1099 \\
Sainte-Rosalie & 1832 & 350 & 200 & 10 & - \\
Saint-Pie & 1828 & 400 & 200 & 90 & 2035 \\
Saint-Charles & 1740 & 450 & 250 & 100 & 1259 \\
Saint-Hyacinthe & 1777 & 500 & 300 & 100 & 3957 \\
Saint-Jean-Baptiste & 1797 & 600 & 250 & 90 & 2143 \\
Saint-Marc & 1792 & 550 & 300 & 150 & 1296 \\
Saint-Antoine & 1750 & 650 & 200 & 150 & 1930 \\
Saint-Damase & 1817 & 800 & 250 & 100 & 2045 \\
Saint-Denis & 1740 & 700 & 400 & 300 & 2863 \\
Beloeil & 1772 & 1100 & 400 & 150 & 1754 \\
\hline
\end{tabular}

Sources: ACAM, RC, 2, Tableau des dîmes des paroisses visitées en 1834; recensement de 1831. 
TABLEAU 5

Moyennes des récoltes paysannes

à La Présentation et Saint-Denis et dîmes des curés vers 1830 (en minots)

\begin{tabular}{lrcc}
\hline & Blé & Avoine & Pois \\
\hline Rangs de La Présentation & & & \\
Salvail & 57 & 77 & 24 \\
Sainte-Rose & 55 & 74 & 20 \\
Les Étangs & 41 & 22 & 10 \\
Grand Rang & 84 & 101 & 26 \\
Saint-Simon & 117 & 113 & 29 \\
\hline Saint-Denis & 137 & 83 & 29 \\
\hline Dîmes (moyenne des 13 paroisses) & 500 & 229 & 104 \\
\hline
\end{tabular}

Sources: Recensement de 1831; Allan Greer, op. cit., 189; ACAM, RC, 2, Tableau des dîmes des paroisses visitées en 1834 .

car les habitants refusèrent par la suite d'acquitter cette charge ${ }^{45}$. Point de tension entre maints pasteurs et leurs paroissiens, le paiement des dîmes était notamment aléatoire dans les terroirs où les habitants vivaient pauvrement ${ }^{46}$. Les poursuites en justice contre les mauvais payeurs étaient déconseillées par l'épiscopat. Par contre, ceux qui payaient mal pouvaient être privés de l'absolution ${ }^{47}$.

En dépit des difficultés pour la percevoir, la dîme procurait à la plupart des curés un revenu nettement supérieur à la production moyenne paysanne. Il suffit, pour s'en convaincre, d'observer les récoltes des habitants de La Présentation en 1831 (tableau 5). Cette paroisse était formée de rangs aux sols très fertiles (le Grand Rang et Saint-Simon), mais comprenait aussi des zones marécageuses moins propices à l'agriculture (Salvail, Sainte-Rose, Les Étangs). Les moyennes obtenues pour chacun des rangs sont inférieures à la dîme moyenne. Seulement 6 habitants sur 181 récoltaient 300 minots de blé ou plus, un seuil atteint par tous les curés, à l'exception d'un seul. La récolte moyenne dans la prospère paroisse de Saint-Denis, qu'Allan Greer a calculée pour 1831, était également inférieure aux dîmes des curés.

45 AESH, XVII, c.35, lettre d'Odelin à Panet, 16 juillet 1832.

46 Voir Thomas Wien, loc. cit., 186.

47 AAQ, RL, 4, 93, lettre de Denaut à Lanctôt, 30 mars 1802. 
Que représentait la dîme en argent? À titre indicatif, reportonsnous aux prix des grains sur différents marchés du Bas-Canada ${ }^{48}$. En 1788 , la dîme de Saint-Hyacinthe dépassait 2000 livres ${ }^{49}$; les grains perçus par le curé de Saint-Denis valaient plus de 3500 livres. En 1834, toutes les paroisses, hormis Saint-Césaire, fournissaient une redevance d'au moins 2000 livres. La valeur des dîmes de SaintCharles, Saint-Marc, Saint-Hyacinthe et Saint-Jean-Baptiste oscillait entre 3000 et 4000 livres. Ce revenu s'élevait même à 6700 livres à Belœil ${ }^{50}$. Les binages autorisaient par ailleurs un cumul des dîmes. En 1820-1821, Pierre Robitaille percevait cette redevance à SaintCharles, à Saint-Marc, à Belœil et à Saint-Hilaire. Au début des années 1830, Antoine Girouard cumulait quant à lui les revenus de Saint-Hyacinthe et de Sainte-Rosalie. Ainsi s'expliquent les protestations de quelques prêtres, instruits du désir de l'évêque de les décharger d'une communauté voisine ${ }^{51}$ ou de diviser leur paroisse ${ }^{52}$.

Ces revenus élevés étaient semblables à ceux des autres paroisses du district de Montréal. Vers 1834, la très grande majorité d'entre elles offraient des revenus supérieurs à 2000 livres $^{53}$. Pour l'ensemble

48 Pour le blé, nous avons retenu les prix moyens dans la vallée du Richelieu, soit 3 livres 12 sols en 1788 et 4 livres 17 sols en 1834 . Faute de données pour la valeur de l'avoine et des pois dans cette région, nous nous référons aux prix de Montréal et de Québec (2 livres 15 sols le minot d'avoine, à Québec, en 1788 et 1 livres 13 sols à Montréal en 1834; 5 livres 7 sols le minot de pois, à Montréal en 1834). Voir F. Ouellet, J. Hamelin, R. Chabot, «Les prix agricoles dans les villes et les campagnes du Québec d'avant 1850: aperçus quantitatifs», Histoire sociale/Social History, 15,29 (mai 1982): 95-96, 99-102, 114-115.

49 À moins d'indication contraire, l'unité monétaire utilisée dans les lignes qui suivent est la livre ancien cours (livre de 20 sols).

50 Depuis 1821, le titulaire de cette cure devait remettre un tiers de la dîme à $\mathrm{Mgr}$ Lartigue, mais celui-ci éprouva des difficultés à faire payer cette redevance. La même règle s'appliquait à Saint-Hilaire, paroisse desservie par le curé de Belœil jusqu'en 1831. À cette date, l'installation d'un curé résidant entraîna la disparition de la pension sur les revenus de SaintHilaire.

51 En 1826, le curé Robitaille de Saint-Charles écrivit à Mgr Plessis pour le prier de lui laisser la desserte de Saint-Marc. La réponse de l'évêque dut le décevoir. Celui-ci lui fit savoir que les revenus d'une seule paroisse étaient suffisants. AAQ, RL, 13, 31, 20 octobre 1826.

52 Le curé Antoine Girouard de Saint-Hyacinthe exprima à plusieurs reprises ses réticences à l'égard des démembrements de sa paroisse. En 1827, il fit même signer une requête contre la création de Saint-Dominique, alléguant que ses revenus diminueraient de moitié. Isidore Desnoyers, Histoire de la paroisse de Saint-Dominique, document conservé aux AESH, 18.

53 Parmi celles-ci, 7 ont des revenus inférieurs à 2000 livres, 33 paient de 2000 à 5000 livres, 15 rapportent plus de 5000 livres. Les paroisses de Boucherville, Longueuil, SainteMarie-de-Monnoir et Varennes, comprises dans cette dernière catégorie, paient annuellement une dîme de 1200,1300 et 1400 minots de blé (auxquels s'ajoutent les autres grains et les pois). Les missions indiennes de Sault-Saint-Louis et de Saint-Régis constituent des cas particuliers. L'argent versé par le gouvernement et, à Saint-Régis, les montants payés par les chefs, complètent les revenus de la dîme. Relevés des dîmes tirés des RAPQ (1942-1943): 157; (19431944): 225, 270, 303; (1944-1945): 204. 
du Bas-Canada, la plupart des cures procuraient également un revenu supérieur à 2400 livres $(£ 100)^{54}$. En proportion, on trouvait toutefois un plus grand nombre de paroisses moins lucratives dans les districts de Québec et de Trois-Rivières.

À la dîme s'ajoutait le casuel. En cette matière, les tarifs différaient d'une paroisse à une autre. Une réglementation visant à instaurer l'uniformité dans la perception des honoraires avait été émise par Mgr Plessis en 1813, mais son application dans le diocèse ne fut pas immédiate ${ }^{55}$. Quatre ans après la publication de ce tarif, l'évêque déplorait encore les excès commis ici et là:

Il y a beaucoup d'abus dans le district de Montréal sur la perception des honoraires. Chacun exige ce qu'il lui plait, sans égard aux anciens règlemens et sans délicatesse de conscience, mais quelquefois avec beaucoup de mauvaise édification pour les fidèles ${ }^{56}$.

Dans la décennie 1840, des différences entre les paroisses subsistaient toujours ${ }^{57}$.

Au début des années 1830, les montants recueillis à titre de casuel s'échelonnaient de 230 à 1200 livres (tableau 6). Le tarif en vigueur et l'importance de la population peuvent expliquer ces différences. Les épidémies, comme le choléra en 1832 et 1834, entraînant un nombre accru de sépultures, influençaient également l'importance du casuel. Moins élevé que les dîmes, celui-ci constituait un revenu d'appoint.

\section{Les interventions économiques des curés}

Bien que variables d'une paroisse à l'autre, les revenus des curés du Richelieu-Yamaska, étaient, à une exception près, confortables. La majorité d'entre eux investirent ces capitaux dans divers champs de l'activité économique.

L'achat d'une ou de plusieurs terres constituait le placement le plus courant. Dans les paroisses, la fabrique mettait à la disposition

54 La Minerve, 19 octobre 1837: 80 curés reçoivent de $£ 75$ à $£ 100,50$ ont de $£ 100$ à $£ 150,30$ ont de $£ 150$ à $£ 200$ et 10 ont de $£ 250$ à $£ 350$.

55 Archives du Séminaire de Québec, Polygraphie 12, no 15. Essai de tarif de Mgr Plessis, 11 février 1813. En 1823, Saint-Césaire était la seule paroisse du Richelieu-Yamaska qui avait adopté ce tarif.

56 AAQ, RL, 9, 300, Plessis à P. Bourget, 31 décembre 1817.

57 «Pour l'uniformité, il serait beaucoup à désirer qu'il y eut un même tarif pour toutes les paroisses, afin que les droits de l'Église fussent moins odieux pour la comparaison qui se fait souvent de ceux qui s'exigent dans les diverses églises de ce diocèse». "Circulaire au clergé, 16 février 1843», Mandements, lettres pastorales, circulaires et autres documents publiés dans le diocèse de Montréal depuis son érection (Montréal, J. Chapleau \& fils, 1887), 1: 233. 
TABLEAU 6

Le casuel dans les paroisses du Richelieu-Yamaska vers 1834 (en livres de 20 sols)

\begin{tabular}{|l|r|}
\hline La Présentation & 75 \\
Sainte-Rosalie & 150 \\
Saint-Charles & 230 \\
Saint-Césaire & 300 \\
Saint-Marc & 300 \\
Saint-Damase & 300 \\
Saint-Jean-Baptiste & 400 \\
Saint-Pie & 400 \\
Saint-Hilaire & 600 \\
Saint-Antoine & 1000 \\
Beloeil & 1000 \\
Saint-Hyacinthe & 1000 \\
Saint-Denis & 1200 \\
Saint-Dominique & - \\
\hline
\end{tabular}

Source: ACAM, RC, 1, État des paroisses visitées en 1834.

des curés quelques arpents de terre qu'ils faisaient cultiver à leur profit. L'usufruit de bâtiments de ferme leur était également réservé. En plus de cette terre, au moins 29 des 49 curés possédaient, en leur nom propre, une ou plusieurs terres dans la région du RichelieuYamaska $^{58}$. L'un d'eux, Antoine Girouard, curé de Saint-Hyacinthe, avait, à son décès en 1832, 1403 arpents $^{59}$. De ces propriétés, les unes cultivées et affermées, les autres non défrichées, il retirait des revenus qui lui permettaient de subventionner le collège qu'il avait fondé. Ses possessions immobilières furent évaluées, dans l'inventaire, à 198000 livres. Édouard Crevier, son successeur à Saint-Hyacinthe, acquit également des terres qu'il donna ensuite aux Sœurs de la Charité afin de les aider à soutenir l'hôpital qu'il avait fondéco ${ }^{60}$ De son côté, le curé

58 Selon un dépouillement des minutes des notaires de la région effectué par le groupe de recherche sur la notabilité rurale de l'Université de Montréal. Nous tenons à remercier Christian Dessureault qui a mis ces documents à notre disposition.

59 Inventaire après décès d'Antoine Girouard, 27 août 1832, selon la transcription faite par J.-A. Dubreuil, procureur du séminaire, 24 mars 1910, Archives de la Société historique de Saint-Hyacinthe, section A, série A, dossier 1.1. L'original, déposé dans le greffe du notaire D.-G. Morisson, a été égaré.

60 AESH, XVII, c.68, Acte de donation en faveur de l'Hôtel-Dieu de Saint-Hyacinthe, 6 février 1840. La donation s'élevait à 67600 livres, hôpital compris. Le document est toutefois ambigu quant à la provenance de 270 arpents (évalués à 27000 livres). Il laisse sous-entendre que ces terres ont été données au curé par des paroissiens désireux de l'aider à soutenir l'œuvre. 
Robitaille de Saint-Charles avait fait l'acquisition de 80 arpents dans sa paroisse et de 2000 acres (2 368 arpents) dans le canton d'Acton ${ }^{61}$. À la fin du XVIII ${ }^{e}$ siècle, François Noiseux acheta aussi plusieurs terres qu'il revendit ensuite avec profit ${ }^{62}$. Ce prêtre se chargea également de la gestion de la seigneurie de Rouville, entre 1780 et 1795 , et de celle de Saint-Hyacinthe, de 1779 à 1796.

L'achat de biens fonciers n'était pas le seul moyen d'investissement des curés. À la fin des années 1830, Édouard Crevier louait, pour $£ 20$ (480 livres ancien cours) par année, la brasserie qu'il possédait au village de Saint-Hyacinthe ${ }^{63}$. Louis Payet, curé de Saint-Antoine, avait quant à lui opté pour la construction de moulins. En 1794, il se vit cependant obligé de légitimer cette initiative, peu commune chez les clercs. Sa lettre, qui laisse sous-entendre le train de vie élevé de plusieurs prêtres, confirme la propension du clergé à investir dans les biens fonciers:

Ces édifices sont les fruits de mes épargnes car pour les ériger, j'ai économisé ce que bien d'autres consumment sur leur table, et par des équipages somptueux, j'avois cru qu'il n'y avait aucune différence entre le produit d'un moulin et celuy d'une terre cultivée. combien entre nos meilleurs curés manquent de fermes? (sans parler des séminaires et des communautés) votre grandeur le scait mieux que moy. quoiqu'il en soit, je vous assure, Monseigneur, que mon temporel ne gêne en rien mon ministêre. j'ai des affidés qui peuvent se passer de mon secour dans le soin de mes propriétés ${ }^{64}$.

Enfin, quelques curés trouvèrent dans le crédit un placement avantageux. Le prêt à intérêt était interdit par l'Église. Dans la majorité des cas, ils eurent donc recours aux constitutions de rentes, une forme de prêt tolérée par les autorités cléricales. Le curé Noiseux de Belœil fut sans contredit le plus notoire de ces prêteurs. Entre 1785 et $1805^{65}$, il avança quelque 120000 livres en argent comptant à ses 158 emprunteurs ${ }^{66}$. Ses créanciers, très majoritairement des habitants, s'engageaient à lui verser annuellement une pension représentant $5 \%$ ou $6 \%$

61 ANQM, minute René Boileau, no 8016, inventaire après décès de Pierre Robitaille, 1er septembre 1834 .

62 Voir Pierre Lambert, Les origines de Beloil (Montréal, Éditions du Méridien, 1991), 106-115.

63 ANQM, minute Dessureau, no 2448, bail à loyer d'Édouard Crevier à Michel Hyacinthe Bellerose, 8 février 1839.

64 AESH, XVII, c.11, lettre de Payet à Hubert, 28 décembre 1794.

65 Il quitta Belœil pour Trois-Rivières en 1796, mais continua de réaliser des opérations financières dans la région du Richelieu-Yamaska.

66 ANQM, minutes Dutalmé, Leguay, Letestu et Mondelet. 
du capital prêté. Michel Vallée, curé de Saint-Marc, conclut des contrats semblables avec plusieurs de ses paroissiens en 1810. Les sommes avancées totalisaient 12900 livres. D'autres curés encore consentirent des constitutions de rentes, mais pour des montants moins importants. Antoine Girouard accorda même quelques obligations comportant un taux d'intérêt de $6 \%{ }^{67}$.

\section{Le niveau de fortune des curés}

Aux revenus liés aux fonctions pastorales s'ajoutaient ainsi les bénéfices que pouvaient rapporter le lopin de la fabrique, les propriétés foncières que les curés possédaient, le crédit et, moins fréquemment, les investissements dans les activités de transformation. En plus de toucher ces revenus, les pasteurs avaient à leur disposition un logement ${ }^{68}$. Il leur restait en somme peu de dépenses à encourir. Comme tout chef de famille, ils devaient défrayer les coûts inhérents à l'alimentation et à l'entretien de leur maisonnée. Ils devaient également payer le vicaire, lorsqu'il y en avait un. À la fin du XVIII ${ }^{e}$ siècle, ces prêtres touchaient annuellement 200 livres, une somme modique en regard des revenus curiaux. Au début du siècle suivant, ce salaire fut majoré par les curés, puis fixé à 300 livres par Mgr Lartigue, en $1824^{69}$. Certains recevaient davantage, mais le supplément était alors payé par la fabrique ${ }^{70}$. Autres frais pour les curés, les gages mensuels d'une ménagère, qui avoisinaient 10 à 15 livres $^{71}$, n'étaient pas de nature à ruiner l'employeur, d'autant plus qu'une parente - souvent une sœur ou la mère - pouvait remplir gratuitement cette fonction. Pour deux prêtres, l'achat d'esclaves avait même résolu temporairement la question de la domesticité ${ }^{72}$.

67 Inventaire après décès d'Antoine Girouard, op. cit.

68 Les disputes au sujet des presbytères sont nombreuses. Les coûts sont sans cesse discutés. À Saint-Hyacinthe, le grand vicaire, prié par l'évêque de faire enquête, accuse le curé d'avoir voulu se construire un palais. Certains curés, las des oppositions, préférèrent payer euxmêmes le presbytère. AESH, XVII, c.25, lettre de Cherrier à Plessis, 31 janvier 1808; AESH, XVII, c.35, lettre d'Odelin à Lartigue, 23 février 1834; ACAM, RC, 1, Ordonnances pour la paroisse de La Présentation visitée les 21,22 et 23 mai 1823. I. Desnoyers, Histoire de la paroisse Saint-Hyacinthe, document manuscrit conservé aux AESH.

69 AAQ, RL, 12, lettre de Plessis à Lartigue, 27 juillet 1824 .

70 En 1835, Mgr Signay incita la fabrique de Saint-Hyacinthe à voter un supplément pour le vicaire, comme cela se pratiquait à Québec et à Trois-Rivières. Les émoluments habituels étaient jugés insuffisants pour «un prêtre assistant le Curé dans le service d'une paroisse populeuse». L'évêque souhaitait que le vicaire touche $£ 50$ (1 200 livres ancien cours). AAQ, RL, 16, lettre de Signay à Crevier, 9 février 1835. passifs.

71 Ce sont les montants mentionnés dans les inventaires après décès, au chapitre des

72 Il s'agit de Louis Payet, curé de Saint-Antoine de 1786 à 1798, et de Pierre Fréchette, curé de Belœil de 1796 à 1816. AESH, XVII, c.11, lettre de Payet à Hubert, 28 octobre 1794. Voir aussi P. Lambert, op. cit., 116. 
Les revenus des curés et les avantages dont ils bénéficiaient dans les paroisses laissent donc entrevoir une vie aisée. Les inventaires après décès de dix d'entre eux ${ }^{73}$ ont permis d'apprécier leur fortune et de la comparer avec celle des individus appartenant aux autres catégories socioprofessionnelles de la seigneurie de Saint-Hyacinthe ${ }^{74}$. Soulignons toutefois que les immeubles n'ont pas été pris en compte dans nos résultats, car leur valeur était absente de la plupart des inventaires.

Dans la hiérarchie des fortunes, les curés occupaient une place enviable (tableau 7). Leurs actifs étaient supérieurs à ceux de tous les autres groupes. Seuls quelques marchands les surpassaient sur ce plan. La fortune des curés se distinguait cependant de celle des marchands, en partie constituée de stocks et de créances difficiles à percevoir. Elle se caractérisait par une forte proportion de biens meubles attestant leur situation de rentiers. Un mobilier important, généralement de qualité, était disposé dans les presbytères ${ }^{75}$. Les miroirs, l'argenterie, les objets de cristal et de porcelaine, les bibelots de faïence se retrouvaient dans la plupart des intérieurs des curés. Quelques-uns possédaient des meubles de marbre et d'acajou et consommaient des denrées importées, notamment du vin, du chocolat et des tissus. À leur décès, leurs avoirs étaient généralement vendus à l'encan. Le produit de la vente allait à des parents, mais quelques curés favorisèrent les fabriques, les institutions d'enseignement et les hôpitaux ${ }^{76}$. Antoine Girouard légua tous ses biens à Mgr Lartigue, qui agissait pour la corporation du collège Saint-Hyacinthe. La plupart des testaments comprenaient aussi des legs aux pauvres de la paroisse. Ils leur laissaient leurs linges et vêtements ou des montants variant entre 300 et 1300 livres $^{77}$.

73 Ces curés étaient, à une exception près, d'origine modeste. Un seul avait moins de 50 ans à son décès. Le plus âgé avait 79 ans. Sept d'entre eux comptaient 18 ans et plus de service dans la région. 379.

74 Les données qui ont servi à cette comparaison proviennent de C. Dessureault, loc. cit.,

75 Voir Christian Dessureault, «Niveau de vie dans le Richelieu-Yamaska, 1800-1840. Étude préliminaire pour une comparaison France-Québec», Gérard Bouchard et Joseph Goy, dir., Famille, économie et société rurale en contexte d'urbanisation (17'-20 siècle), avec la collaboration de J.-P. Wallot, J. Dickinson, J. Roy (Chicoutimi et Paris, Centre interuniversitaire SOREP et École des hautes études en sciences sociales, 1990), 185-198. Actes du colloque d'histoire comparée Québec-France, tenu à Montréal en février 1990.

76 Tous les curés de la région, décédés entre 1790 et 1840 , ont fait un testament.

77 ANQM, minute De la Grave, testament de Pierre Fréchette, 11 décembre 1845; minute Perrin, no 480, testament de Bonaventure Alinotte, 4 décembre 1834. Voir aussi C.-P. Choquette, Histoire du Séminaire de Saint-Hyacinthe depuis sa fondation jusqu'à nos jours (Montréal, Imprimerie des Sourds-Muets, 1911), 1: 145. 
TABLEAU 7

Fortune mobilière et groupes socioprofessionnels

Richelieu-Yamaska 1795-1839

(en livres de 20 sols)

\begin{tabular}{|c|c|c|c|c|c|c|c|c|c|}
\hline Groupes sociaux & $\begin{array}{c}\text { Biens de } \\
\text { consommation }\end{array}$ & $\begin{array}{l}\text { Biens de } \\
\text { production }\end{array}$ & Cheptel & Stocks & Numéraire & Argenterie & Créances & Actifs & Passifs \\
\hline $\begin{array}{l}\text { Curés } \\
\text { (1800-1839) } \\
\text { Marchands }\end{array}$ & 3964 & 417 & 1583 & 664 & 573 & 324 & 6543 & 14048 & 6079 \\
\hline $\begin{array}{r}(1795-1814) \\
(1825-1834)\end{array}$ & $\begin{array}{r}881 \\
1550\end{array}$ & $\begin{array}{l}282 \\
373\end{array}$ & $\begin{array}{l}1159 \\
1044\end{array}$ & $\begin{array}{l}1705 \\
2099\end{array}$ & - & $\begin{array}{l}18 \\
95\end{array}$ & $\begin{array}{r}4561 \\
30680\end{array}$ & $\begin{array}{r}8606 \\
35680\end{array}$ & $\begin{array}{l}14783 \\
24376\end{array}$ \\
\hline $\begin{array}{c}\text { Professions libéra } \\
(1795-1814) \\
(1825-1834)\end{array}$ & $\begin{array}{r}206 \\
1305\end{array}$ & $\begin{array}{r}98 \\
147\end{array}$ & $\begin{array}{l}137 \\
694\end{array}$ & $\begin{array}{r}7 \\
180\end{array}$ & - & $\overline{181}$ & - & $\begin{array}{r}448 \\
2929\end{array}$ & $\begin{array}{l}1758 \\
3429\end{array}$ \\
\hline $\begin{array}{l}\text { Artisans } \\
(1795-1814) \\
(1825-1834)\end{array}$ & $\begin{array}{l}322 \\
282\end{array}$ & $\begin{array}{l}134 \\
138\end{array}$ & $\begin{array}{l}216 \\
230\end{array}$ & $\begin{array}{l}192 \\
295\end{array}$ & $\begin{array}{r}1 \\
51\end{array}$ & - & $\begin{array}{l}351 \\
435\end{array}$ & $\begin{array}{ll}1 & 206 \\
1 & 431\end{array}$ & $\begin{array}{ll}1722 \\
1683\end{array}$ \\
\hline $\begin{array}{l}\text { Cultivateurs } \\
(1795-1814) \\
(1825-1834)\end{array}$ & $\begin{array}{l}204 \\
210\end{array}$ & $\begin{array}{l}104 \\
122\end{array}$ & $\begin{array}{l}348 \\
444\end{array}$ & $\begin{array}{l}330 \\
346\end{array}$ & $\begin{array}{l}37 \\
22\end{array}$ & - & $\begin{array}{l}203 \\
341\end{array}$ & $\begin{array}{ll}1226 \\
1485\end{array}$ & $\begin{array}{r}737 \\
1182\end{array}$ \\
\hline $\begin{array}{l}\text { Journaliers } \\
(1795-1814) \\
(1825-1834)\end{array}$ & $\begin{array}{l}54 \\
78\end{array}$ & $\begin{array}{l}37 \\
44\end{array}$ & $\begin{array}{l}34 \\
77\end{array}$ & $\begin{array}{l}43 \\
11\end{array}$ & $\begin{array}{l}25 \\
17\end{array}$ & - & $\begin{array}{l}35 \\
38\end{array}$ & $\begin{array}{l}228 \\
265\end{array}$ & $\begin{array}{l}270 \\
197\end{array}$ \\
\hline
\end{tabular}
Ћ Sources: Les inventaires après décès des curés. C. Dessureault, «Crise ou modernisation? La société maskoutaine durant le premier tiers du XIX e siècle», RHAF,
42,3 (hiver 1989): 370. 


\section{CONCLUSION}

Afin de comprendre l'action du clergé paroissial et ses rapports avec les fidèles, il nous est apparu essentiel de mieux connaître son cheminement professionnel, son niveau de fortune et ses interventions dans l'économie locale. Quatorze paroisses du Richelieu-Yamaska ont servi de terrain d'enquête. Il appert qu'à la fin du XVIII ${ }^{e}$ et au début du $\mathrm{XIX}^{\mathrm{e}}$ siècle, la pénurie de prêtres hâta les carrières cléricales. Les besoins du diocèse permirent à de jeunes prêtres méritants ou entrés dans les bonnes grâces de l'épiscopat de parvenir rapidement à un bénéfice lucratif. Pour la plupart des prêtres, la première nomination à une cure survenait avant l'âge de 30 ans.

Les promotions rapides avaient de multiples conséquences. Elles engendraient des tensions au sein du clergé car les assignations provoquaient le mécontentement et la jalousie. La confraternité sacerdotale avait donc ses limites.

De façon plus générale, les promotions rapides soulèvent des questions sur l'activité pastorale et sociale des curés et sur leurs relations avec les paroissiens. Ainsi, la logique des carrières cléricales entrait en contradiction avec le désir des évêques d'assurer un encadrement efficace. Les curés méritants étaient placés dans les bonnes paroisses, celles où la population était réputée docile et le revenu élevé, alors que plusieurs prêtres tombés en disgrâce, s'ils n'étaient pas nommés vicaires, échouaient dans les missions en périphérie où tout restait à faire. On a vu par ailleurs que de jeunes curés éprouvaient des difficultés à faire cautionner par les paroissiens leurs décisions touchant l'administration locale. Dans quelle mesure l'inexpérience du clergé paroissial s'est-elle répercutée sur son action pastorale? Enfin, il faudrait connaître l'influence de la durée des mandats sur l'encadrement pastoral dispensé. Il était sans doute plus difficile d'établir de façon durable un réseau d'associations et de pratiques de dévotion dans une paroisse qui changeait très fréquemment de curé.

Sur le plan financier, le curé disposait d'un revenu fort appréciable qui faisait de lui un des personnages les plus riches de la paroisse. Même s'il était majoritairement issu de la paysannerie, le clergé paroissial du Richelieu-Yamaska était socialement éloigné de ses paroissiens. Il était doté d'une instruction supérieure. Ses revenus et son rôle économique étaient importants et ont sans doute donné une coloration particulière à son activité et à ses rapports avec les paroissiens. Dispensateurs des sacrements, prédicateurs, gardiens de la moralité publique, des curés étaient aussi les créanciers de leurs paroissiens. L'un d'eux fut même le représentant du pouvoir seigneurial. 
Ces revenus, cette aisance ont peut-être incité quelques garçons ambitieux à opter pour le sacerdoce. Cependant, d'autres facteurs commandaient la vocation ou, au contraire, la décourageaient. Sinon, comment expliquer la pénurie de prêtres de l'après-conquête? L'étude du recrutement sacerdotal, amorcé avec les travaux sur l'origine du clergé, devra désormais se pencher sur ces questions, prendre en considération les différents éléments de la carrière pastorale qui pouvaient inciter au sacerdoce ou en détourner la jeunesse. Elle devra également se pencher sur la ferveur religieuse, sur les perceptions du clergé et de la religion, de même que sur les efforts et les moyens mis en œuvre pour recruter une relève ecclésiastique. 